\title{
Visualizing a protonated RNA state that modulates
}

\section{microRNA-21 maturation}

Jared T. Baisden ${ }^{1}$, Joshua A. Boyer ${ }^{1}$, Bo Zhao ${ }^{1,2}$, \& Qi Zhang ${ }^{1, *}$

${ }^{1}$ Department of Biochemistry and Biophysics, University of North Carolina at Chapel Hill, Chapel Hill, North Carolina, USA

${ }^{2}$ Department of Chemistry, University of North Carolina at Chapel Hill, Chapel Hill, North Carolina, USA

*Correspondence should be addressed to Q.Z. (zhangqi@unc.edu) 


\begin{abstract}
MicroRNAs are evolutionarily conserved small, non-coding RNAs that regulate diverse biological processes. Due to their essential regulatory roles, microRNA biogenesis is tightly regulated, where protein factors are often found to interact with specific primary and precursor microRNAs for regulation. Here, using NMR relaxation dispersion spectroscopy and mutagenesis, we reveal that the precursor of oncogenic microRNA-21 exists as a pH-dependent ensemble that spontaneously reshuffles the secondary structure of the entire apical stem-loop region, including the Dicer cleavage site. We show that the alternative excited conformation transiently sequesters the bulged adenine into a non-canonical protonated $A^{+}-G$ mismatch, conferring a two-fold enhancement in Dicer processing over its ground conformational state. These results indicate that microRNA maturation efficiency may be encoded in the intrinsic dynamic ensemble of primary and precursor microRNAs, providing potential means of regulating microRNA biogenesis in response to environmental and cellular stimuli.
\end{abstract}


MicroRNAs (miRNAs) are highly conserved, small noncoding RNAs that regulate more than $60 \%$ of protein coding genes at the post-transcriptional level ${ }^{1-5}$. Most miRNAs are initially transcribed by RNA polymerase II as introns of protein-coding genes or from independent coding genes into long primary transcripts (pri-miRNAs) that feature 5 '-end 7-methylguanosine caps and $3^{\prime}$-end poly-A tails ${ }^{6,7}$. In the canonical biogenesis pathway, pri-miRNAs are subsequently processed into $\sim 70$ nucleotide precursor hairpins (premiRNAs) by the Microprocessor complex, consisting of one RNase III family enzyme, Drosha, and two DiGeorge critical region 8 proteins (DGCR8) $)^{8,9}$. Pre-miRNAs are then exported from the nucleus to the cytoplasm by Exportin-5 (Ref. 10) and further processed into $\sim 20$ base-pair miRNA/miRNA* duplexes by another RNase III family enzyme, Dicer, in complex with transactivation-responsive RNA binding protein $(\mathrm{TRBP})^{11,12}$. The resulting single-stranded mature miRNA is incorporated into the miRNA-inducing silencing complex (miRISC), which regulates protein expression by repressing translation, promoting deadenylation, and/or cleaving target mRNA ${ }^{13}$.

Due to their essential regulatory roles, miRNA biogenesis is tightly regulated to ensure proper gene expression ${ }^{3-5}$, and abnormal miRNA regulation has often been associated with cancer, neurological disorders, cardiovascular diseases and others ${ }^{14,15}$. Remarkably, despite sharing the same set of enzymes in the canonical biogenesis pathway, individual miRNAs exhibit cell-type and cell-state specific expressions. Even those clustered on the same primary transcript can be differentially processed in a tissue-specific manner ${ }^{3-5}$. Over the past decade, it has been shown that specific sequences and structures of primary and precursor miRNAs can be recognized by processing machineries and protein factors for regulation ${ }^{16-26}$. For example, pri-miRNAs 
that possess a UGU motif in the apical loops are preferentially processed by the Microprocessor ${ }^{19,20}$, pre-miRNAs encoding a two-nucleotide distance between the cleavage sites and the apical bulge/loop structures are more accurately processed by Dicer $^{21}$, and miRNAs that feature stable basal stems in pri-miRNAs and flexible apical loops in pri-/pre-miRNAs are more efficiently processed by biogenesis machineries ${ }^{22}$. In addition, altering secondary structures and even primary sequences of pri-/pre-miRNAs via protein binding ${ }^{23}$, enzymatic-driven nucleotide modification ${ }^{24,25}$ and disease-linked mutation ${ }^{26}$ can further influence the outcome of miRNA biogenesis ${ }^{3}$. During these regulatory processes, it is often perceived that protein factors act on the largely passive primary and precursor miRNAs to direct their maturation outcome. However, despite many non-coding RNAs having been shown to actively explore their conformational dynamics for function ${ }^{27}$, it remains elusive whether pri-/pre-miRNAs can play a role in modulating miRNA biogenesis in the absence of protein factors. This is largely due to our limited high-resolution structural and dynamic knowledge of most pri-/pre-miRNAs, where key regulatory elements of pri-/pre-miRNAs, such as the apical stem-loop region also known as the pre-element region, are often too flexible to be studied by conventional structural biology approaches.

Here, using NMR relaxation dispersion (RD) spectroscopy and Dicer processing assays, we set out to characterize the structural dynamics of microRNA-21 precursor (pre-miR-21) and examine how the intrinsic RNA conformational plasticity may contribute to miRNA maturation. MicroRNA-21, one of the first identified human miRNAs ${ }^{28}$, functions as an oncogene involved in tumorigenesis, progression, metastasis, and cell survival ${ }^{29}$, where its biogenesis is regulated at both transcriptional 
and post-transcriptional levels ${ }^{30}$. Previous studies have shown that the pre-element region of pri-/pre-miR-21 serves as an important element for regulating miR-21 biogenesis. Mutations that stabilize the pre-element inhibit Microprocessor processing of pri-miR-21 (Ref. 31), whereas binding of the KH-type splicing regulatory protein (KSRP) at this location promotes enzymatic processing of pri-/pre-miR-21s ${ }^{32}$. By carrying out NMR RD measurements, we discovered that the pre-element region of pri/pre-miR-21 exists as a pH-dependent ensemble, which undergoes a two-state structural transition and dynamically accesses a low-populated $(\sim 1-15 \%)$ transient, yet kinetically stable (lifetime $\sim 0.8 \mathrm{~ms}$ ) state referred to as an excited state $(E S)$ across physiologically relevant ranges of $\mathrm{pH}(\mathrm{pH} \sim 6.5-8.0)$. With ${ }^{15} \mathrm{~N}$ chemical exchange saturation transfer (CEST) NMR spectroscopy, we were able to directly measure, for the first time, an adenine N1 protonation event, which occurs at the Dicer cleavage site and underlies this unique, $\mathrm{pH}$-dependent structural transition. This adenine protonation corresponds to a concerted secondary structural reshuffling of the entire pre-element region, transitioning the adenine from a bulged residue in the ground-state (GS) conformation to being sequestered into a non-canonical $A^{+}(a n t i)-G(s y n)$ base pair in the ES. We further demonstrated that these distinct structures are processed differently by Dicer, where the ES-mimicking substrate is processed to mature miR-21 with a two-fold enhancement in efficiency over its GS counterpart. Hence, despite adopting an apparently simple secondary structure, pre-miR-21 encodes a dynamic ensemble at its pre-element region that encapsulates environmentally sensitive states with distinct fitness for processing. With the emerging view of RNA ES as a 'hidden' layer for regulation ${ }^{27}$, our results further suggest that miRNA processing intermediates may 
employ ES-encoded dynamic ensembles as potential means to regulate microRNA biogenesis in response to environmental and cellular stimuli.

\section{RESULTS}

The pre-element region of pre-miR-21 samples distinct conformational states

The miR-21 precursor consists of the pre-element region and miR-21/miR-21* helix, and is predicted to fold into a hairpin structure with four double-stranded helices, three bulges, and one apical loop (Fig. 1a). To focus on the pre-element region, we designed a shorter RNA construct, preE-miR-21, which contains the entire pre-element and the adjacent helix from the miR-21/miR-21* stem (Fig. 1b). NMR ${ }^{1} \mathrm{H}-{ }^{1} \mathrm{H}$ NOESY experiment on the imino region provides an excellent characterization of RNA secondary structure, as one imino resonance is expected for a canonical Watson-Crick base pair and two imino resonances are expected for a G-U wobble pair. Except for the formation of Watson-Crick base pairs at the lower stem, the pre-element of miR-21 does not adopt a stable conformation, as only weak imino resonances of G-U wobble pairs can be observed in the NMR ${ }^{1} \mathrm{H}-{ }^{1} \mathrm{H}$ NOESY spectrum (Fig. 1c). This observation is consistent with previous NMR studies on miR-21 precursor ${ }^{33,34}$, where mutations of the pre-element were made to quench the structural flexibility into a single conformational state $^{33}$. When we carried out an NMR ${ }^{13} \mathrm{C}-{ }^{1} \mathrm{H}$ HSQC experiment that probes nonsolvent-exchangeable signals, surprisingly only 20 out of a total of 29 expected NMR resonances from preE-miR-21 were observed at room temperature (Fig. 1c). This spectroscopic behavior resembles the typical NMR phenomenon of exchange broadening, where interconversion between two or more states can lead to the 
disappearance of NMR signals. Indeed, by raising the temperature from $25^{\circ} \mathrm{C}$ to a more physiologically relevant $35^{\circ} \mathrm{C}$, most of the missing resonances reappeared in the NMR ${ }^{13} \mathrm{C}-{ }^{1} \mathrm{H}$ HSQC spectrum (Fig. 1c), confirming the presence of conformational exchange.

Recent developments of NMR $R_{1 p}$ RD spectroscopy have opened new avenues to quantify microsecond-to-millisecond conformational changes and made it possible to study RNA ESs that are too low-populated and short-lived to be detected by conventional techniques ${ }^{35-39}$. Here, we carried out both on-resonance and off-resonance low spin-lock field $R_{1 \rho} R D$ experiments to quantify the exchange process in preE-miR21. For residues from the miR-21/miR-21* stem region, we observed flat RD profiles for base (C2, C5, C6, and C8) and sugar (C1') carbons (Fig.1d and Supplementary Fig. 1), which are consistent with one stable helical conformation of this region. In contrast, residues within the pre-element region, ranging from the bulge residue $A 22$ to the stem residue A35 (Fig. 1d and Supplementary Fig. 2), display power and offset dependent RD profiles. Indeed, these RD profiles can be global-fitted to a single two-state (GS $\leftrightarrow$ ES) exchange process. These results reveal that the pre-element region is not only conformationally flexible, but also dynamically interconverts between at least two structurally and kinetically distinct states, where the ES has a low population ( $\left.p_{\mathrm{ES}}\right)$ of $15.2 \pm 0.3 \%$ and a short lifetime $\left(\tau_{\mathrm{ES}}=1 / \mathrm{k}_{\mathrm{EG}}\right)$ of $816 \pm 15 \mu \mathrm{s}($ Fig. $1 \mathrm{~d})$.

\section{The ES involves transient protonation at the Dicer cleavage site}

To gain structural insights into the ES, we utilized NMR chemical shifts, which are one of the most sensitive measurements for probing local chemical environments. We examined ES carbon chemical shifts $\left(\varpi_{\mathrm{ES}}=\varpi_{\mathrm{GS}}+\Delta \varpi\right)$, where $\Delta \varpi$ is the chemical shift 
difference between ES ( $\left.\varpi_{\mathrm{ES}}\right)$ and $\mathrm{GS}\left(\varpi_{\mathrm{GS}}\right)$ extracted from the two-state analysis of an $\mathrm{R}_{1 \rho} \mathrm{RD}$ profile (Supplementary Fig. 3). Among all the extracted ES chemical shifts, the base carbon $\mathrm{C} 8$ of bulge $\mathrm{A} 22$, which resides at the Dicer cleavage site, displays the largest deviation $(\Delta \varpi)$ with a $\sim 1.49$ ppm down-field shift from its GS position (Fig. 1d and Supplementary Fig. 3). Notably, we were not able to obtain the RD profile for base carbon $\mathrm{C} 2$ of $\mathrm{A} 22$, as the $\mathrm{C} 2 \mathrm{H} 2$ resonance remains severely broadened beyond detection in the NMR ${ }^{13} \mathrm{C}-{ }^{1} \mathrm{H}$ HSQC (Fig. 2a), suggesting even larger perturbations in carbon $\mathrm{C} 2$ and/or proton $\mathrm{H} 2$ chemical shifts between ES and GS. The dramatically different behavior of $\mathrm{C} 8 \mathrm{H} 8$ and $\mathrm{C} 2 \mathrm{H} 2$ resonances from the same base is reminiscent of recent NMR studies on transiently N1-protonated adenines ${ }^{40,41}$.

To examine whether the exchange process could be due to possible protonation events, we increased the $\mathrm{pH}$ of the sample from 6.45 to 8.04 , aiming to shift the equilibrium towards non-protonated states. Indeed, the NMR ${ }^{13} \mathrm{C}-{ }^{1} \mathrm{H} H S Q \mathrm{C}$ spectrum recorded at $\mathrm{pH} 8.04$ exhibits much higher quality, where exchange broadening of most resonances is substantially reduced, such that the $\mathrm{A} 22-\mathrm{C} 2 \mathrm{H} 2$ resonance can be readily observed (Fig. 2a). Low spin-lock field NMR $\mathrm{R}_{1 \rho} \mathrm{RD}$ measurements provide further quantitative support that the observed exchange involves a protonated ES of the preelement region of miR-21 (Fig. 2b and Supplementary Fig. 4). Global fit of RD profiles showed that the GS $\leftrightarrow$ ES equilibrium is significantly shifted towards GS at $\mathrm{pH} 8.04$, where the ES population $\left(p_{\mathrm{ES}}\right)$ is reduced to a mere $1.1 \pm 0.1 \%$. In addition, a two-state analysis of the RD profile of base carbon A22-C2 further revealed a remarkable $7.9 \mathrm{ppm}$ difference between its GS and ES chemical shifts, resulting in an ES chemical shift of 144.5 ppm (Fig. 2b and Supplemental Fig. 5). This significantly up-field shifted C2 
chemical shift is consistent with $\mathrm{C} 2$ chemical shifts reported for stably N1-protonated adenines, strongly suggesting that $\mathrm{A} 22$ is protonated at the $\mathrm{N} 1$ site in the ES.

Recently, we have developed nucleic-acid-specific ${ }^{15} \mathrm{~N}$ CEST NMR spectroscopy to study RNA conformational exchanges using non-proton-bonded nitrogens as probes $^{42}$. This technique also enables direct evaluation of the protonation status of adenines in low-populated and short-lived states, which have remained elusive to date. By measuring ${ }^{15} \mathrm{~N}$ CEST profiles at $\mathrm{pH} 8.04$, we were able to unambiguously identify that $\mathrm{A} 22$ is transiently protonated at N1 (Fig. 2c). Unlike N1 of A30, which is not protonated and displays an apparent single-dip CEST profile, the nitrogen CEST profile of A22-N1 exhibits two distinct intensity dips that correspond to two alternative conformations. A two-state analysis of the CEST profile validates that A22-N1 probes the same two-state exchange process, where extracted ES population $\left(p_{\mathrm{ES}-\mathrm{CEST}} \sim 1.1 \pm\right.$ $0.1 \%)$ and lifetime $\left(\tau_{\text {ES-CEST }} \sim 645 \pm 114 \mu \mathrm{s}\right)$ from ${ }^{15} \mathrm{~N}$ CEST are very similar to those obtained from ${ }^{13} \mathrm{C} \mathrm{R}_{1 \rho} \mathrm{RD}$ at pH $8.04\left(p_{\mathrm{ES}-\mathrm{R} 1 \rho} \sim 1.1 \pm 0.1 \%, \tau_{\mathrm{ES}-\mathrm{R} 1 \rho} \sim 823 \pm 109 \mu \mathrm{s}\right)$. The ES chemical shift of A22-N1 directly supports a protonated N1 with an unprecedented up-field shift of $67.3 \pm 0.2 \mathrm{ppm}$ to $157.7 \mathrm{ppm}$, residing well among resonances of protonbonded imino nitrogens in RNA ( 134-152 ppm in Gs and 154-165 ppm in Us).

To obtain more insights into the $A 22(G S) \leftrightarrow A 22^{+}(E S)$ transition, we further carried out carbon $\mathrm{R}_{1 \rho} \mathrm{RD}$ and nitrogen CEST measurements on base $(C 2, \mathrm{C} 8, \mathrm{~N} 1)$ and sugar (C1') moieties of A22 at pH 6.96 and 7.47 (Fig. 2d and Supplementary Fig. 5). Consistent with being a protonation-dependent process, the population of $\mathrm{A} 22^{+}$ gradually increases from $\sim 1 \%$ at $\mathrm{pH} 8.04$ to $\sim 15 \%$ at $\mathrm{pH} 6.45$. Surprisingly, the lifetime of the ES A22+ remains largely unperturbed between $\mathrm{pH} 6.45$ and $\mathrm{pH} 8.04$, where the 
average lifetime is $\tau_{E S} \sim 847 \pm 49 \mu$ s (Fig. $2 e$ ). In contrast, the apparent lifetime of the GS, which is derived from the extracted rate of exchange $\left(\tau_{G S}=1 / k_{G E}\right)$, reduces substantially from $\tau_{\mathrm{GS}} \sim 74 \mathrm{~ms}$ at $\mathrm{pH} 8.04$ to $\tau_{\mathrm{GS}} \sim 5 \mathrm{~ms}$ at $\mathrm{pH} 6.45$ (Fig. 2e). The high population of $\mathrm{A}_{22} 2^{+}$at $\mathrm{pH} 6.45$, which otherwise would be close to zero based on the intrinsic $\mathrm{pK}_{\mathrm{a}}(\sim 3.5)$ of free adenine $\mathrm{N} 1$ site $^{43}$, further suggests that $\mathrm{A} 22$ has a distinct protonation propensity when compared with other unstructured adenines. Consistent with this observation, $\mathrm{pH}$-dependent chemical shift analyses showed a $\mathrm{pK}_{\mathrm{a}}$ value of 5.84 \pm 0.08 for $\mathrm{A} 22$, which is substantially shifted towards neutral $\mathrm{pH}$ from adenines in the apical-loop that have an average $\mathrm{pK}_{\mathrm{a}}$ value of $4.17 \pm 0.06$ (Fig. $2 \mathrm{f}$ and Supplementary Fig. 6). Taken together, these results unambiguously revealed that preE-miR-21 undergoes a $\mathrm{pH}$-dependent conformational transition, where A22 at the Dicer cleavage site is specifically protonated in the ES.

\section{The transient protonation couples global secondary structural reshuffling}

How does the excited state stabilize a site-specific protonation? To address this, we first evaluated the role of each structural motif of the pre-element region - the bulge, the stem, and the apical loop - in the observed conformational transition (Fig. 3a-C and Supplementary Fig. 7). Bulge A22 is the site of protonation. Without A22, we could not detect any conformational exchange within the rest of the pre-element region, as evidenced with flat RD profiles for the A22-deletion mutant (Fig. 3a). Not only is a protonated A22 the result of the structural transition, this protonation may likely be the chemical basis that triggers the larger transition across the entire pre-element region. In addition to the indispensable bulge A22, we found that both a weak stem and a flexible 
apical loop are needed to achieve the structural transition. Stabilizing the two G-U wobble pairs with G-C Watson-Crick pairs completely quenches the exchange (Fig. 3b); replacing the apical loop with a highly structured UUCG tetraloop also eliminates the transition (Fig. 3c). These results suggest that the pre-element region serves as a unified structural entity to enable a concerted transition towards stabilizing the protonated excited state. The rate of exchange $\left(k_{\mathrm{ex}}=k_{\mathrm{GE}}+k_{\mathrm{EG}} \sim 1445 \mathrm{~s}^{-1}\right)$ is an order of magnitude slower than rates observed from local structural changes involving transient adenine protonation ${ }^{44}$, but similar to the secondary-structure-based long-range communication observed HIV-1 TAR RNA ${ }^{45}$, further supporting a global secondary structural reshuffling of the pre-element region.

To further delineate the secondary structure of the excited state, we employed a mutate-and-chemical-shift-fingerprinting strategy ${ }^{44}$. In this approach, mutations are introduced to stabilize conformational features unique to a proposed/predicted ES secondary structure, which are then validated by comparing chemical shift differences between the mutant and wild-type ( $\left.\Delta \varpi_{\mathrm{mut}}\right)$ to those extracted from NMR RD profiles $(\Delta \varpi \mathrm{RD})$. Here, we used $\mathrm{MC}$-fold ${ }^{46}$ to predict possible alternative low-energy secondary structures of preE-miR-21. Strikingly, most of the predicted structures share a common feature of an A22-G38 base pair, whereas the remaining pre-element adopts various secondary structures that are distinct from the ground-state conformation (Supplementary Fig. 8). Being protonated at N1, A22 could potentially be base paired with $\mathrm{G} 38$ in the $\mathrm{ES}$, albeit in the $\mathrm{A}^{+}($anti)-G(syn) form rather than the conventional $A(a n t i)-G(a n t i)$ pair, where the syn conformation of $G 38$ is indicated with the down-field chemical shift of base carbon $\mathrm{C} 8\left(\Delta \varpi_{E S}=2.6 \mathrm{ppm}\right)$ at $\mathrm{pH} 8.04$ (Supplementary Fig. 4). 
An interesting structural feature of the $A^{+}(a n t i)-G(s y n)$ pair is that it largely retains an overall A-form-like geometry with an inter-sugar distance of $10.4 \AA^{47}$, whereas the $\mathrm{A}\left(\right.$ anti)-G(anti) pair substantially widens this distance to $12.9 \AA^{48}$ and subsequently distorts the helical geometry of neighboring base pairs (Fig. 3d).

To test this proposed ES structural feature, we mutated G38 to a uridine, which not only sequesters A22 into a base pair, but also maintains an A-form geometry at the site of mutation. The G38U mutant converges into a single state as evidenced by flat RD profiles (Fig. 3e and Supplementary Fig. 9), and largely represents the ES of preE-miR21 based on chemical shifts. Good agreement was observed between $\Delta \varpi_{\mathrm{G}} 38 \mathrm{U}$ and $\Delta \varpi_{\mathrm{RD}}$ for 15 out of 19 base and sugar carbon resonances from the pre-element residues with detectable RD profiles, including A22(C1'), C23(C1'), G28(C1'), A29(C2/C8), A30(C8), U31(C1'), C32(C1'), U33(C6), C34(C6), A35(C1'/C2/C8), U36(C6), and C39(C5) (Fig. 3f and Supplementary Figs. 3 and 4). The agreement of sugar carbon C1's of A22 and C23 further supports the ES adopting an A-form-like backbone geometry at the site of protonation. The deviations between $\Delta \varpi_{G} 38 \mathrm{U}$ and $\Delta \varpi_{\mathrm{RD}}$ for base carbons $\mathrm{C} 2$ and $\mathrm{C} 8$ of A22 can be attributed to protonation-induced major chemical shift perturbations in the wild-type, which cannot be recapitulated with this mutation. However, the deviations for C32-C5 and U36-C1' could be due to their relatively small chemical shift differences $(<0.5 \mathrm{ppm})$ and/or local conformational perturbations in the wild-type from the mutation, which is subject to future investigation. As the G38U mutant closely mimics the ES, it also provides some insights into the two residues (U24 and U26) in the pre-element region that showed no detectable RD. For U24-C6, its flat RD profile can be explained with essentially identical chemical shifts between the ground and excited states as 
indicated with $\Delta \varpi_{G} 38 \mathrm{U} \sim 0$, whereas the lack of detectable RD for U26-C6 may be due to additional local conformational perturbations that are subject to further studies (Supplementary Fig. 9). To provide independent validation of the chemical shift fingerprints of the ES, we compared $\Delta \varpi \mathrm{G} 38 \mathrm{U}$ to chemical shift differences of the wild type between $\mathrm{pH} 8.04$ and $\mathrm{pH} 4.81\left(\Delta \varpi_{\mathrm{pH}}\right)$, where the low $\mathrm{pH}$ value was chosen to shift the population towards the protonated ES without inducing global protonation of adenines and cytosines. Good agreement was observed between $\Delta \varpi_{R D}$ and $\Delta \varpi_{p H}$, except for some major deviations from unpaired adenines and cytosines that are likely due to rapid protonation at $\mathrm{pH} 4.81$ given their intrinsic $\mathrm{pK}_{\mathrm{a}}(\sim 3.5-4.2)^{43}$ when unpaired (Fig. $3 f$ and Supplementary Figs. 3 and 4). In particular, excellent agreement between $\Delta \varpi_{R D}$ and $\Delta \varpi_{\mathrm{pH}}$ of $\mathrm{A} 22-\mathrm{C} 2$ and $\mathrm{A} 22-\mathrm{C} 8$ complements the G38U mutant. Taken together, low $\mathrm{pH}$ and G38U are each able to recapitulate a portion of the ES structure, with low pH chemical shifts matching changes in A22 residues, and G38U chemical shifts matching throughout the rest of the structure. These results strongly suggest that pre-miR-21 undergoes a global structural reshuffling at the pre-element region, where A22 is transiently protonated and forms a distinct $A^{+}($anti)-G(syn) base pair in the ES.

\section{The miR-21 precursor encodes states with differential Dicer processivities}

The conformational transition of pre-miR-21 is the protonation-driven base pairing of the bulged A22 that resides specifically at the location of Dicer cleavage. Hence, it is of interest to see how these structural changes may affect Dicer cleavage of the miR-21 precursor. To examine this, we designed GS- and ES-mimicking substrates and performed processing assays using commercially available recombinant human 
Dicer (Fig. 4a). For the GS-mimicking substrate (pre-miR-21GS), we mutated the two GU wobbles with two $\mathrm{G}-\mathrm{C}$ pairs, which was shown to stabilize the flexible stem that forms in the ground state. For the ES-mimicking structure (pre-miR-21 ${ }^{\mathrm{ES}}$ ), we incorporated a G38U mutation to the full-length pre-miR-21. We would like to note that the G38U mutant, which recapitulates key ES structural features, cannot perfectly mimic the electrostatic property of the protonated ES. However, since Dicer cleaves the phosphate backbone, we anticipate structures of the backbone, rather than the base pairing identity of the ES, may influence Dicer activity. In order to generate a native-like precursor with 5'-terminal phosphate group and sequence, we fused a hammerhead ribozyme to the 5'-end of full-length miR-21 precursor. During in vitro transcription, the hammerhead ribozyme self-cleaves, and the resulting miR-21 precursor is 5'-end phosphorylated and labelled with [ $\gamma^{-32}$ P] ATP. Remarkably, GS- and ES-mimicking substrates exhibited substantially different Dicer processivities (Fig. 4b). As can be seen, $\sim 23 \pm 6 \%$ of pre-miR-21 ${ }^{\mathrm{GS}}$ was cleaved by Dicer to generate mature miR- 21 , which is essentially identical to a processivity of $\sim 26 \pm 9 \%$ for the wild-type substrate (pre-miR-21 ${ }^{\mathrm{WT}}$ ). This is consistent with pre-miR-21 ${ }^{\mathrm{WT}}$ occupying $\sim 99 \%$ GS under the assay condition of $\mathrm{pH}$ 8.04. In contrast, Dicer processed pre-miR-21ES much more efficiently than its GS counterpart, where double the amount of substrate ( $48 \pm 6 \%)$ was converted to mature miR-21. Together, these results not only unveil differential fitness of the GS and ES of the pre-element region of the pre-miR-21 for miR-21 maturation, but further exemplify the importance of RNA structures in directing the overall outcome of miRNA biogenesis. 


\section{DISCUSSION}

Here, by integrating structural, dynamic, and functional analyses on miR-21 precursor, we showed that the intrinsic conformational plasticity of miRNA processing intermediates can serve as a new layer of regulation for miRNA biogenesis. Often, structural changes in pri-/pre-miRNAs can be induced upon binding to protein regulators ${ }^{23}$, nucleotide modifications such as ADAR1-mediated adenine-to-inosine editing $^{24}$ and METTL1-mediated methylation ${ }^{25}$, and disease-linked mutations ${ }^{26}$. RNA structural motifs are also important factors in recognition by processing machineries for miRNA biogenesis, where altering primary and/or precursor structures of a target miRNA can further lead to altered biogenesis, inducing a change in physiological outcomes $^{3}$. In contrast to these adaptive changes, we found that pre-miR-21 encodes a dynamic ensemble in its apical stem-loop region that undergoes spontaneous structural transitions between two kinetically and functionally distinct states (Fig. 5). In the ground state, the two Dicer cleavage sites reside within a largely unstructured region; in contrast, both locations become structured in the excited state. Relative to the ground state, the excited state conformation more closely resembles the optimal structure for Dicer, where both cleavage sites are base-paired and positioned two-nucleotides away from a flexible apical loop ${ }^{21}$, hence, providing a better topology for Dicer cleavage.

A hallmark of the ES of pre-miR-21 is protonation of the adenine residue at the Dicer cleavage site. Protonation is a fundamental chemical property and one of the smallest chemical modifications on nucleic acids ${ }^{43}$. The intrinsic $\mathrm{pK}_{a} \mathrm{~s}$ for protonation of adenines and cytosines are acidic and reside far from the physiological $\mathrm{pH}$ ranges. However, by adopting sophisticated structures, RNA can shift acidic $\mathrm{pK}_{\mathrm{a}} \mathrm{s}$ toward neutral 
pHs, such that specific ionization can be achieved under physiological conditions for function $^{43}$. For example, the universally conserved adenine residue at the active site of the ribosome has a shifted $\mathrm{pK}_{\mathrm{a}}$ to serve as a general acid-base catalyst for peptide formation ${ }^{49}$, whereas the murine leukemia virus (MLV) recoding signal employs a protonated adenine as a structural factor to stabilize a compact pseudoknot in order to allow the ribosome to bypass the Gag stop codon ${ }^{40}$. Here, protonation provides the crucial chemical basis for pre-miR-21 to form the $A^{+}(a n t i)-G(s y n)$ base pair, which ensures the cleavage site adopts an overall A-form-like topology in the ES. Without being protonated, the adenine residue may still be able to pair with the upper stem guanine residue, albeit in the form of $A(a n t i)-G(a n t i)$ mismatch, which is likely functionally indistinguishable from the GS. Another feature of the protonation event in pre-miR-21 is that the underlying structural transition occurs at the millisecond timescale, which is substantially faster than those involved in major structural changes, such as the adenine protonation in MLV40. This fast GS $\leftrightarrow$ ES interconversion could enable pre-miR-21 to rapidly reach new equilibrium upon a transient high acid load due to disease-induced metabolic shifts, modulating maturation of miR-21 in response to environmental stimuli. Despite displaying distinct in vitro outcomes, a functional understanding of the role of pre-miR-21 protonation in regulating biogenesis will require future investigations that evaluate the response of pre-miR-21 under various cellular conditions such as hypoxia and acidosis.

Interestingly, the spontaneous conformational transition in pre-miR-21, which involves secondary structural reshuffling of the pre-element region, is reminiscent of that observed in Lin28-dependent regulation of the biogenesis of let-7 family of miRNAs ${ }^{50}$. In 
general, RNA secondary structural changes encounter large kinetic barriers, hence, need to be catalyzed by external factors such as RNA-binding proteins. Both domains of Lin28 work cooperatively to bind two independent RNA elements to induce the regulatory structural changes of pri-/pre-let-7s. In contrast, pre-miR-21 accomplishes such structural changes without protein factors by accessing an excited state, where the bulged adenine base pairs with the upper stem guanine residue, propagating a global change in strand register. Here, all three structural elements of the apical stem-loopthe bulge, metastable stem, and flexible loop - are essential to achieve this concerted movement, and eliminating any of them abolishes the spontaneous transitions in premiR-21. While our observation represents the first example of an excited state in premiRNAs, an ES-based mechanism for remodeling distant motifs has also been recently reported in HIV-1 TAR RNA ${ }^{45}$. Since the apical stem-loop is a common structural feature among all pri-/pre-miRNAs, we speculate that many miRNA processing intermediates may encode similar ES-based conformational plasticity for long-range communication across their regulatory pre-element regions, which is further supported by a recent computational modeling of secondary structural ensembles of miRNAs ${ }^{26}$. It has become increasingly clear that many non-coding RNAs (ncRNAs) do not fold into single static structures, instead, they dynamically interconvert between different conformational states for function ${ }^{27}$. Recent developments in NMR techniques have opened new avenues to probe RNA structural transitions involving alternative conformational states that often evade detection from conventional biophysical and biochemical methods due to their low populations and/or transient lifetimes ${ }^{38,39,44}$. Indeed, these technical advances have unveiled the presence of a diverse set of excited 
states in non-coding RNAs. Our discovery of the transiently protonated state in pre-miR21 was also made possible by these new techniques. In particular, the development of a nucleic-acid-optimized ${ }^{15} \mathrm{~N}$ NMR CEST method has enabled, for the first time, direct identification of transient protonation in nucleic acids, illuminating the crucial chemical basis for delineating the relationship between structure and function. With the emerging view of ESs as a 'hidden' layer of regulation ${ }^{27}$, the growing repertoire of functional RNA ESs with distinct structural features promise novel strategies and developments in RNAtargeted therapeutics.

\section{METHODS}

Methods, including statements of data availability and references, are available in the online version of the paper.

\section{Acknowledgements}

We thank G. Young and S. Parnham for maintenance of NMR instruments and members of the Zhang lab for critical comments. This work was supported by start-up fund from the University of North Carolina at Chapel Hill and an NSF grant (MCB1652676).

\section{Author contributions}

J.T.B. and Q.Z. conceived the project and experimental design. J.T.B., J.A.B., B.Z. and Q.Z. prepared the samples, carried out NMR experiments, analyzed the data, and wrote the paper.

\section{Competing financial interests}

The authors declare no competing financial interests. 
bioRxiv preprint doi: https://doi.org/10.1101/852822; this version posted November 23, 2019. The copyright holder for this preprint (which was not certified by peer review) is the author/funder. All rights reserved. No reuse allowed without permission.

\section{Additional information}

Any supplementary information, chemical compound information and source data are available in the online version of the paper. Reprints and permissions information is available online at http://www.nature.com/reprints/index.html. Correspondence and requests for materials should be addressed to Q.Z. 


\section{REFERENCES}

1. Friedman, R. C., Farh, K. K., Burge, C. B. \& Bartel, D. P. Most mammalian mRNAs are conserved targets of microRNAs. Genome Res. 19, 92-105 (2009).

2. Siomi, H. \& Siomi, M. C. Posttranscriptional regulation of microRNA biogenesis in animals. Mol. Cell 38, 323-332 (2010).

3. Ha, M. \& Kim, V. N. Regulation of microRNA biogenesis. Nat. Rev. Mol. Cell Biol. 15, 509-524 (2014).

4. Gebert, L. F. R. \& MacRae, I. J. Regulation of microRNA function in animals. Nat. Rev. Mol. Cell Biol. 20, 21-37 (2019).

5. Treiber, T., Treiber, N. \& Meister, G. Regulation of microRNA biogenesis and its crosstalk with other cellular pathways. Nat. Rev. Mol. Cell Biol. 20, 5-20 (2019).

6. Lee, Y. et al. MicroRNA genes are transcribed by RNA polymerase II. EMBO J. 23, 4051-4060 (2004).

7. Cai, X., Hagedorn, C. H. \& Cullen, B. R. Human microRNAs are processed from capped, polyadenylated transcripts that can also function as mRNAs. RNA 10, 1957-1966 (2004).

8. Lee, Y. et al. The nuclear RNase III Drosha initiates microRNA processing. Nature 425, 415-419 (2003).

9. Han, J. et al. The Drosha-DGCR8 complex in primary microRNA processing. Genes Dev. 18, 3016-3027 (2004).

10. Yi, R., Qin, Y., Macara, I. G. \& Cullen, B. R. Exportin-5 mediates the nuclear export of pre-microRNAs and short hairpin RNAs. Genes Dev. 17, 3011-3016 (2003). 
11. Hutvagner, G. et al. A cellular function for the RNA-interference enzyme Dicer in the maturation of the let-7 small temporal RNA. Science 293, 834-838 (2001).

12. Ketting, R. F. et al. Dicer functions in RNA interference and in synthesis of small RNA involved in developmental timing in C. elegans. Genes Dev. 15, 2654-2659 (2001).

13. Krol, J., Loedige, I. \& Filipowicz, W. The widespread regulation of microRNA biogenesis, function and decay. Nat. Rev. Genet. 11, 597-610 (2010).

14. Esteller, M. Non-coding RNAs in human disease. Nat. Rev. Genet. 12, 861-874 (2011).

15. Lin, S. \& Gregory, R. I. MicroRNA biogenesis pathways in cancer. Nat. Rev. Cancer 15, 321-333 (2015).

16. Zhang, X. \& Zeng, Y. The terminal loop region controls microRNA processing by Drosha and Dicer. Nucleic Acids Res. 38, 7689-7697 (2010).

17. Tsutsumi, A., Kawamata, T., Izumi, N., Seitz, H. \& Tomari, Y. Recognition of the pre-miRNA structure by Drosophila Dicer-1. Nat. Struct. Mol. Biol. 18, 1153-1158 (2011).

18. Castilla-Llorente, V., Nicastro, G. \& Ramos, A. Terminal loop-mediated regulation of miRNA biogenesis: selectivity and mechanisms. Biochem. Soc. Trans. 41, 861-865 (2013).

19. Auyeung, V. C., Ulitsky, I., McGeary, S. E. \& Bartel, D. P. Beyond secondary structure: primary-sequence determinants license pri-miRNA hairpins for processing. Cell 152, 844-858 (2013). 
20. Nguyen, T. A., Park, J., Dang, T. L., Choi, Y. G. \& Kim, V. N. Microprocessor depends on hemin to recognize the apical loop of primary microRNA. Nucleic Acids Res. 46, 5726-5736 (2018).

21. Gu, S. et al. The loop position of shRNAs and pre-miRNAs is critical for the accuracy of dicer processing in vivo. Cell 151, 900-911 (2012).

22. Contrant, M. et al. Importance of the RNA secondary structure for the relative accumulation of clustered viral microRNAs. Nucleic Acids Res. 42, 7981-7996 (2014).

23. van Kouwenhove, M., Kedde, M. \& Agami, R. MicroRNA regulation by RNAbinding proteins and its implications for cancer. Nat. Rev. Cancer 11, 644-656 (2011).

24. Liu, Z., Wang, J., Li, G. \& Wang, H. W. Structure of precursor microRNA's terminal loop regulates human Dicer's dicing activity by switching DExH/D domain. Protein Cell 6, 185-193 (2015).

25. Pandolfini, L. et al. METTL1 Promotes let-7 MicroRNA Processing via m7G Methylation. Mol. cell 74, 1278-1290 e1279 (2019).

26. Dallaire, P. et al. Structural dynamics control the MicroRNA maturation pathway. Nucleic Acids Res. 44, 9956-9964 (2016).

27. Ganser, L. R., Kelly, M. L., Herschlag, D. \& Al-Hashimi, H. M. The roles of structural dynamics in the cellular functions of RNAs. Nat. Rev. Mol. Cell Biol. 20, 474-489 (2019).

28. Zeng, Y. \& Cullen, B. R. Sequence requirements for micro RNA processing and function in human cells. RNA 9, 112-123 (2003). 
29. Volinia, S. et al. A microRNA expression signature of human solid tumors defines cancer gene targets. Proc. Natl. Acad. Sci. USA 103, 2257-2261 (2006).

30. Kumarswamy, R., Volkmann, I. \& Thum, T. Regulation and function of miRNA-21 in health and disease. RNA Biol. 8, 706-713 (2011).

31. Zeng, Y., Yi, R. \& Cullen, B. R. Recognition and cleavage of primary microRNA precursors by the nuclear processing enzyme Drosha. EMBO J. 24, 138-148 (2005).

32. Trabucchi, M. et al. The RNA-binding protein KSRP promotes the biogenesis of a subset of microRNAs. Nature 459, 1010-1014 (2009).

33. Chirayil, S., Wu, Q., Amezcua, C. \& Luebke, K. J. NMR characterization of an oligonucleotide model of the miR-21 pre-element. PloS One 9, e108231 (2014).

34. Shortridge, M. D. et al. A macrocyclic peptide ligand binds the oncogenic microRNA-21 precursor and suppresses Dicer processing. ACS Chem. Biol. 12, 1611-1620 (2017).

35. Massi, F., Johnson, E., Wang, C., Rance, M. \& Palmer, A. G., 3rd. NMR R1 rho rotating-frame relaxation with weak radio frequency fields. J. Am. Chem. Soc. 126, 2247-2256 (2004).

36. Korzhnev, D. M., Orekhov, V. Y. \& Kay, L. E. Off-resonance R(1rho) NMR studies of exchange dynamics in proteins with low spin-lock fields: an application to a Fyn SH3 domain. J. Am. Chem. Soc. 127, 713-721 (2005).

37. Hansen, A. L., Nikolova, E. N., Casiano-Negroni, A. \& Al-Hashimi, H. M. Extending the range of microsecond-to-millisecond chemical exchange detected 
in labeled and unlabeled nucleic acids by selective carbon R(1rho) NMR spectroscopy. J. Am. Chem. Soc. 131, 3818-3819 (2009).

38. Sekhar, A. \& Kay, L. E. NMR paves the way for atomic level descriptions of sparsely populated, transiently formed biomolecular conformers. Proc. Natl. Acad. Sci. USA 110, 12867-12874 (2013).

39. Palmer, A. G., 3rd. Chemical exchange in biomacromolecules: past, present, and future. J. Magn. Reson. 241, 3-17 (2014).

40. Houck-Loomis, B. et al. An equilibrium-dependent retroviral mRNA switch regulates translational recoding. Nature 480, 561-564 (2011).

41. Chu, C. C., Plangger, R., Kreutz, C. \& Al-Hashimi, H. M. Dynamic ensemble of HIV-1 RRE stem IIB reveals non-native conformations that disrupt the Revbinding site. Nucleic Acids Res. 47, 7105-7117 (2019).

42. Zhao, B., Baisden, J. T. \& Zhang, Q. Probing excited conformational states of nucleic acids by nitrogen CEST NMR spectroscopy. J. Magn. Reson. doi: https://doi.org/10.1016/j.jmr.2019.106642 (2019).

43. Thaplyal, P. \& Bevilacqua, P. C. Experimental approaches for measuring pKa's in RNA and DNA. Methods Enzymol. 549, 189-219 (2014).

44. Dethoff, E. A., Petzold, K., Chugh, J., Casiano-Negroni, A. \& Al-Hashimi, H. M. Visualizing transient low-populated structures of RNA. Nature 491, 724-728 (2012).

45. Lee, J., Dethoff, E. A. \& Al-Hashimi, H. M. Invisible RNA state dynamically couples distant motifs. Proc. Natl. Acad. Sci. USA 111, 9485-9490 (2014). 
46. Parisien, M. \& Major, F. The MC-Fold and MC-Sym pipeline infers RNA structure from sequence data. Nature 452, 51-55 (2008).

47. Pan, B., Mitra, S. N. \& Sundaralingam, M. Crystal structure of an RNA 16-mer duplex R(GCAGAGUUAAAUCUGC)2 with nonadjacent G(syn).A+(anti) mispairs. Biochemistry 38, 2826-2831 (1999).

48. Leonard, G. A. et al. Crystal and molecular structure of r(CGCGAAUUAGCG): an RNA duplex containing two G(anti).A(anti) base pairs. Structure 2, 483-494 (1994).

49. Muth, G. W., Ortoleva-Donnelly, L. \& Strobel, S. A. A single adenosine with a neutral pKa in the ribosomal peptidyl transferase center. Science 289, 947-950 (2000).

50. Nam, Y., Chen, C., Gregory, R. I., Chou, J. J. \& Sliz, P. Molecular basis for interaction of let-7 microRNAs with Lin28. Cell 147, 1080-1091 (2011). 


\section{FIGURE LEGENDS}

Figure 1 NMR characterization of preE-miR-21. (a) Secondary structure of pre-miR21 with dicer cleavage sites highlighted as scissors. (b) Secondary structure of preEmiR-21 construct derived from NMR data, where Watson-Crick base pairs, GU wobbles, and potential Watson-Crick base pairs are highlighted with lines, filled circles, and open circles, respectively. (c) NMR ${ }^{1} \mathrm{H}-{ }^{1} \mathrm{H}$ NOESY spectrum of the imino proton region of preE-miR-21 at $10^{\circ} \mathrm{C}$ and ${ }^{13} \mathrm{C}-{ }^{1} \mathrm{H}$ HSQC spectra of base carbon (C6 and $\mathrm{C} 8$ ) region of preE-miR-21 at $25^{\circ} \mathrm{C}$ and $35^{\circ} \mathrm{C}$. (d) Representative ${ }^{13} \mathrm{C}$ on-resonance and offresonance relaxation dispersion (RD) profiles at $35^{\circ} \mathrm{C}$ showing dependence of $R_{2}+R_{\mathrm{ex}}$ on spin-lock power ( $\left.\omega_{\text {eff }} / 2 \pi\right)$ and offset $(\Omega / 2 \pi)$, respectively, where $\Omega$ is the difference between the spin-lock carrier frequency and the observed resonance frequency. RD profiles of A40 are fit to a single-state model and RD profiles of A22 and A35 are fit to a global two-state model using the Bloch-McConnell equation. Error bars are experimental uncertainties (s.d.) estimated from mono-exponential fitting of $n=3$ independently measured peak intensities.

\section{Figure 2 PreE-miR-21 populates a protonated excited state with a neutral shifted} pKa. (a) NMR ${ }^{13} \mathrm{C}-{ }^{1} \mathrm{H}$ HSQC spectra of base carbon (C2, C6, and C8) region of preEmiR-21 at pH 6.45 and $\mathrm{pH}$ 8.04. (b) ${ }^{13} \mathrm{C}$ off-resonance $\mathrm{RD}$ profiles of $\mathrm{A} 22-\mathrm{C} 2$ at $\mathrm{pH} 8.04$. (c) ${ }^{15} \mathrm{~N}$ CEST profiles of $\mathrm{A} 30-\mathrm{N} 1$ and $\mathrm{A} 22-\mathrm{N} 1$ at $\mathrm{pH} 8.04$, which are fit to a single-state and a two-state model, respectively, using the Bloch-McConnell equation. (d) The $\mathrm{pH}-$ dependent ${ }^{13} \mathrm{C}$ off-resonance RD profiles of $\mathrm{A} 35-\mathrm{C} 8$ at spin-lock power of $\omega_{\text {efff }} / 2 \pi=299$ Hz. (e) The pH-dependent apparent lifetimes of GS and ES from RD analysis. (f) The $\mathrm{pH}$-dependent population of the excited state based on $\mathrm{R}_{1 \rho} \mathrm{RD}$ data and A22-C8 
chemical shift (CS) for extracting an apparent $\mathrm{pK}_{\mathrm{a}}$ of $\mathrm{A} 22$. Representative $\mathrm{pK}_{\mathrm{a}}$ derived from unpaired A29, A30 and A35 is shown in black. Error bars are experimental uncertainties (s.d.) estimated from mono-exponential fitting of $n=3$ independently measured peak intensities.

Figure 3 Excited-state structure of preE-miR-21. (a-c) Secondary structures and representative ${ }^{13} \mathrm{C}$ on-resonance and off-resonance $\mathrm{RD}$ profiles of bulge, stem, and loop mutants. (d) Sugar-sugar (C1'-C1') distances of A-U Watson-Crick base pair, A-G mismatch, and $A^{+}-G$ mismatch. (e) Secondary structure and representative ${ }^{13} \mathrm{C}$ onresonance and off-resonance RD profiles of ES-mimic mutant. (f) Comparison of carbon chemical shifts for the GS, ES, the mutant mimics, and wild-type construct at $\mathrm{pH} 4.81$. Error bars are experimental uncertainties (s.d.) estimated from mono-exponential fitting of $n=3$ independently measured peak intensities.

Figure 4 Excited state of preE-miR-21 enhances dicer processing. (a) Secondary structures of the wild-type, ground-state mimic, and excited-state mimic of pre-miR-21. (b) Dicer processing assays of wild-type and mutant pre-miR-21s, quantification using ImageQuant shown on right $\left({ }^{* *}\right.$ denotes $p \leqslant 0.01$ via student T-Test). Shown are means and standard deviations (s.d.) from $n=4$ independent assays.

\section{Figure 5 Modulation of miR-21 maturation with a protonation-dependent}

structural ensemble. Protonation at N1 site of A22 sequesters the bulged adenine into a non-canonical $A^{+}-G$ mismatch and is associated with a long-range conformational 
reshuffling of the pre-element region. This structural rearrangement results in a conformation that is better suited for Dicer processing to generate mature miR-21. 


\section{Figure 1}
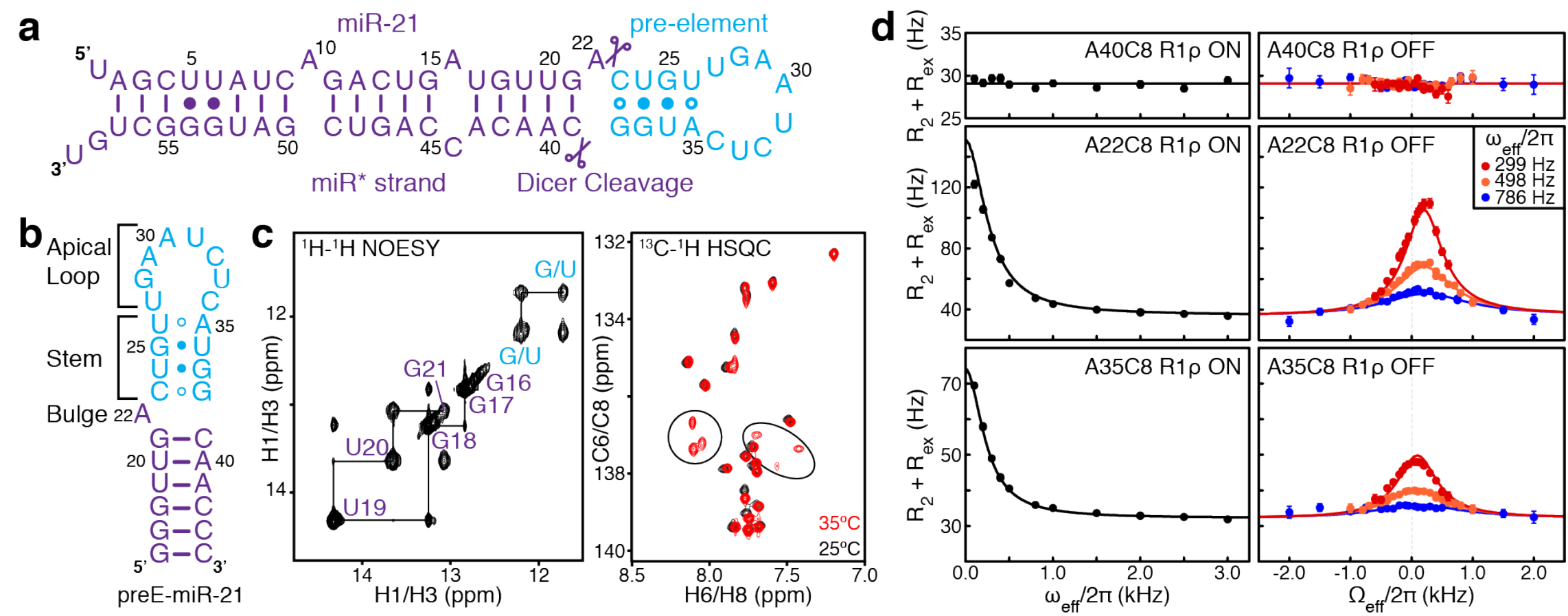
Figure 2
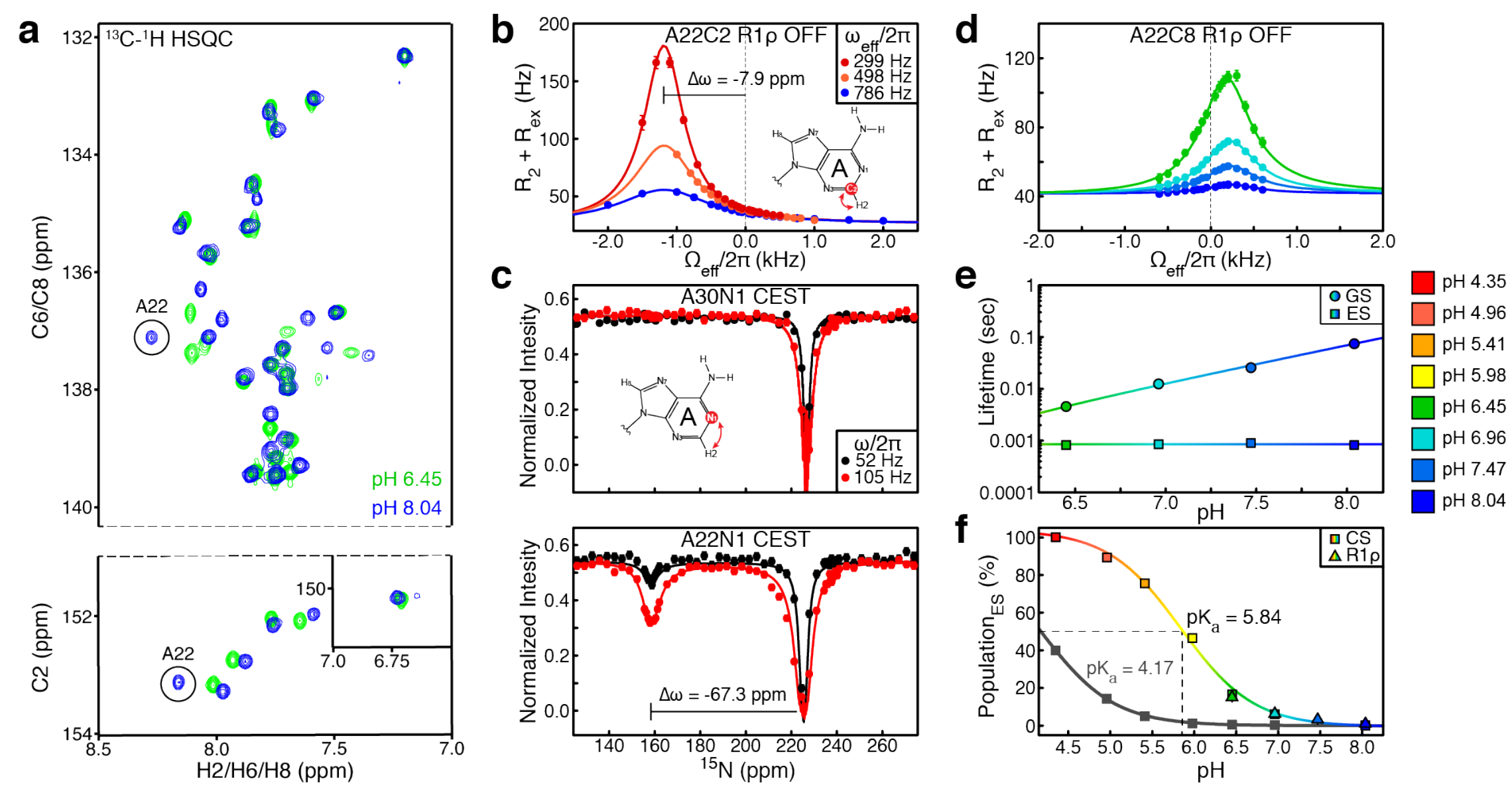

Page 30 


\section{Figure 3}

a

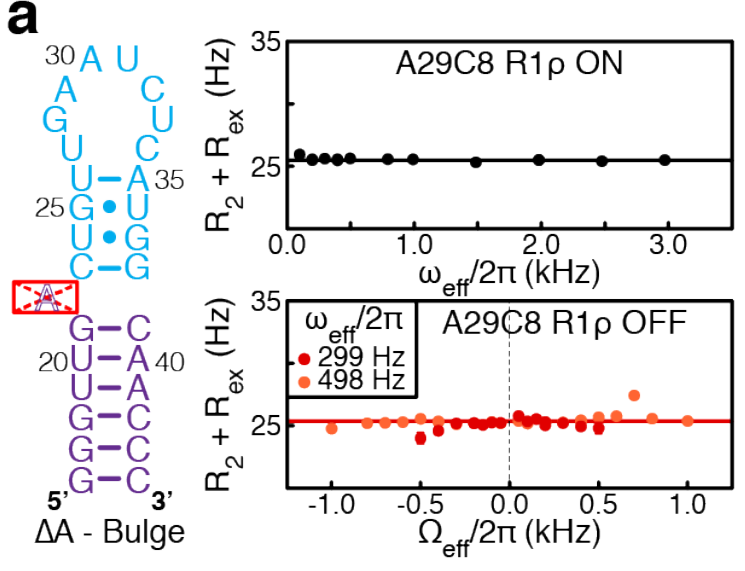

b

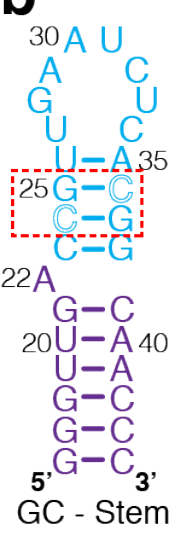

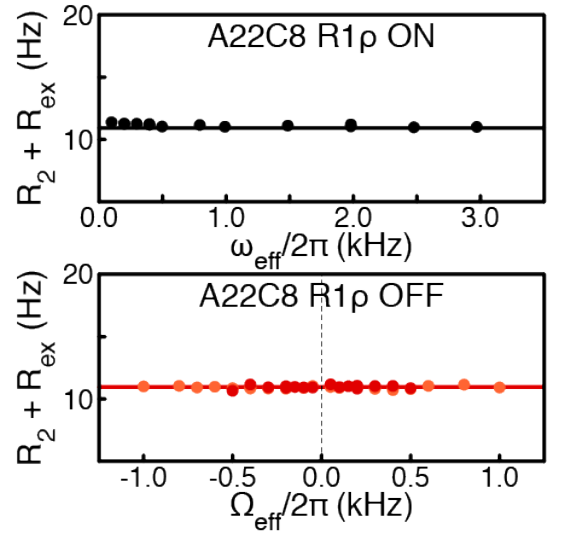

C

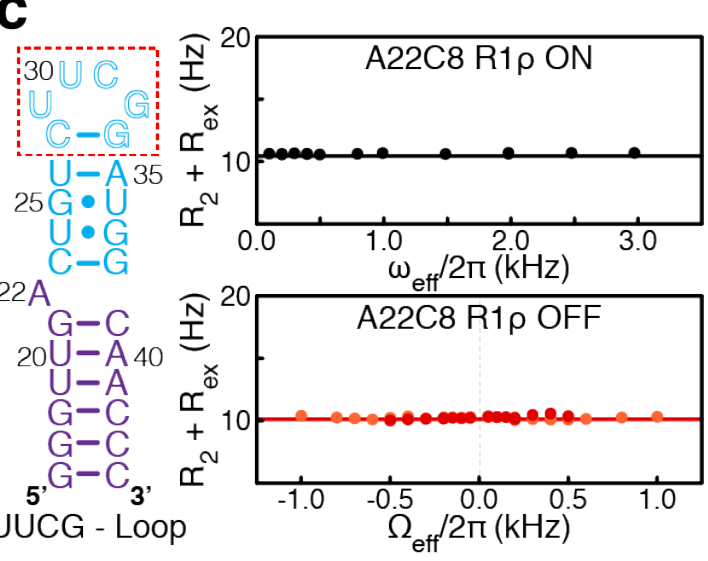

d

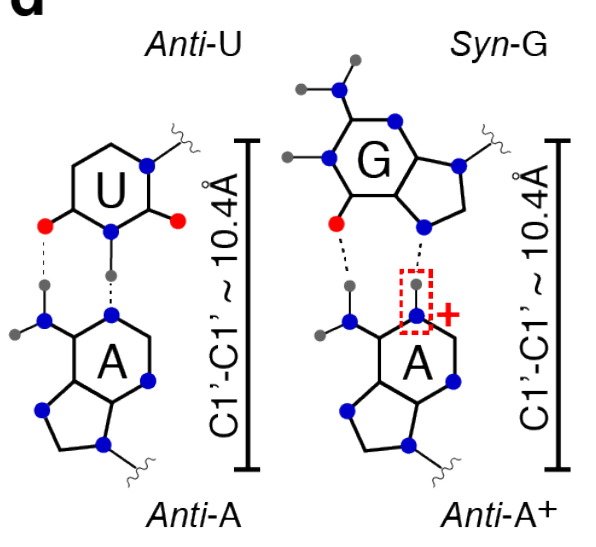

e

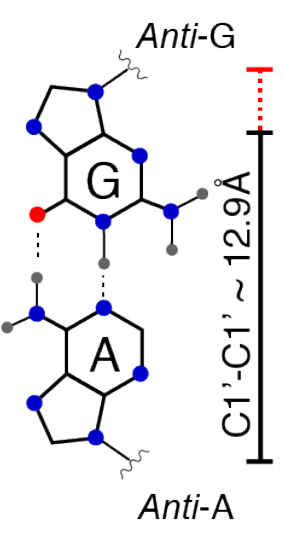

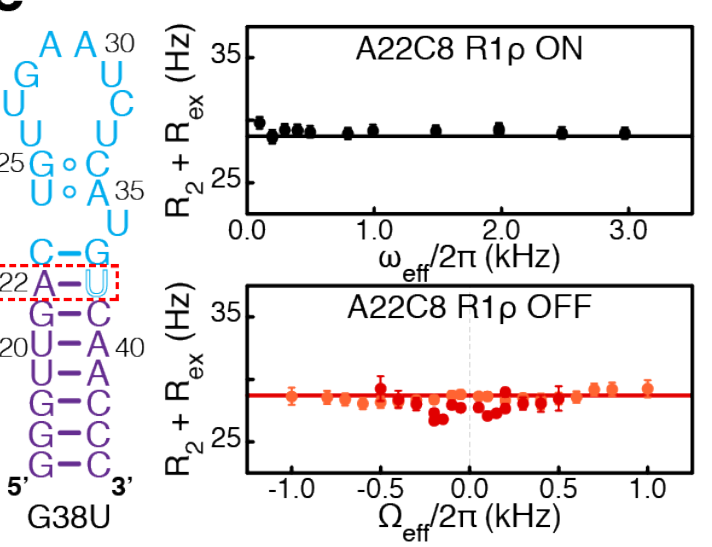

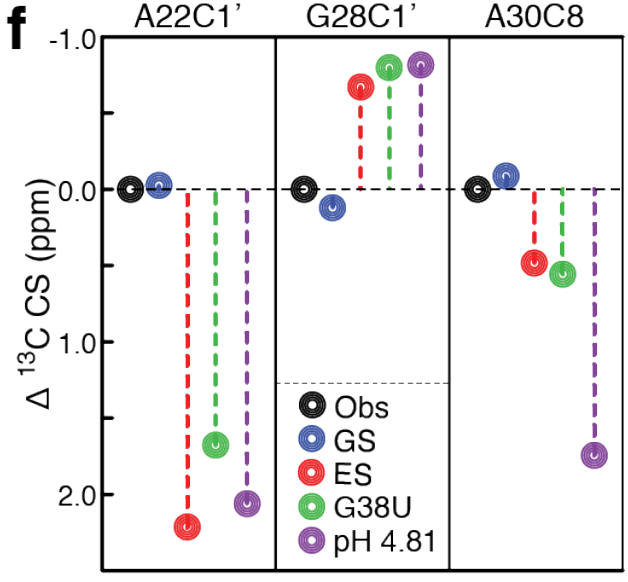




\section{Figure 4}

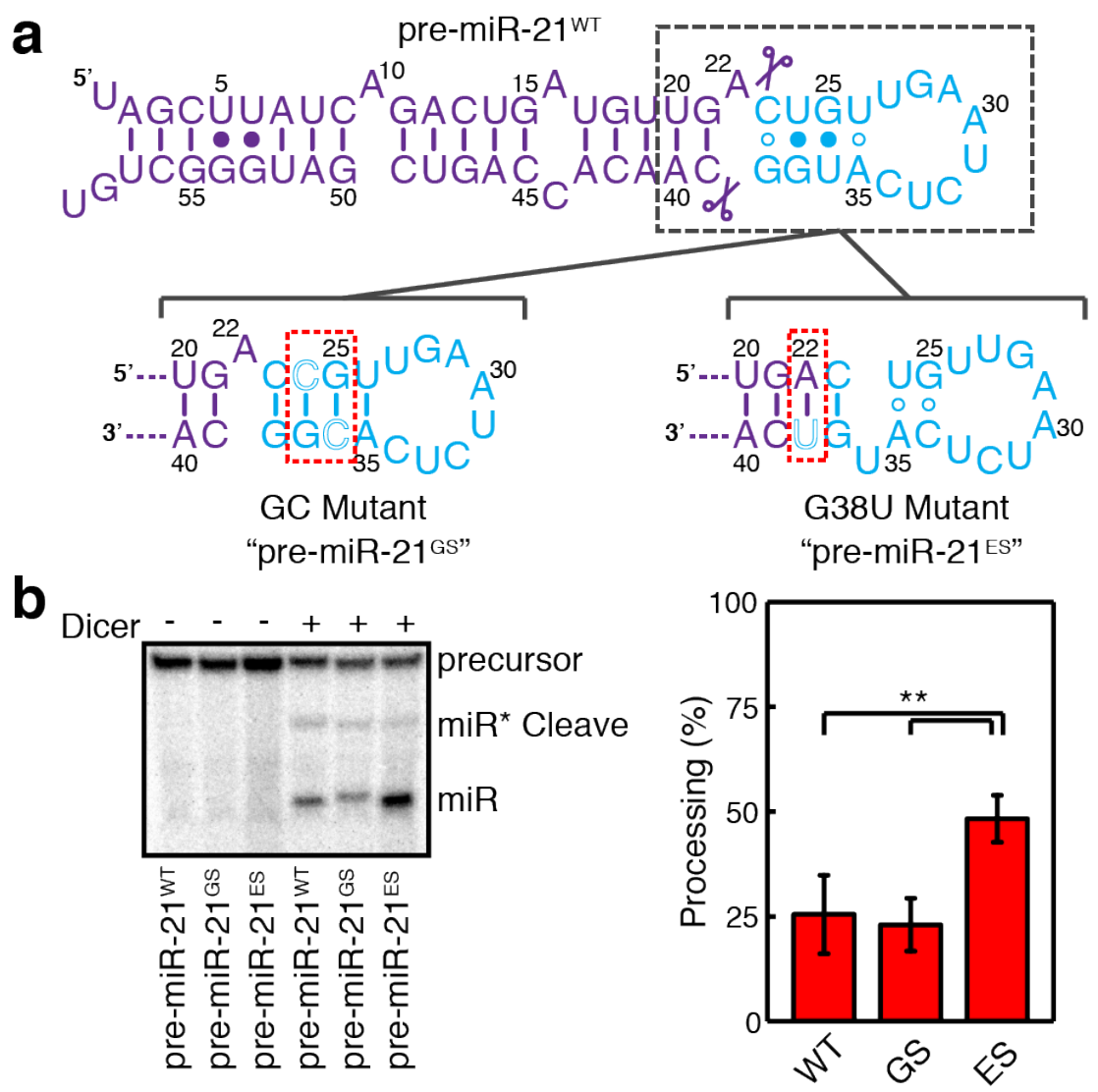


Figure 5

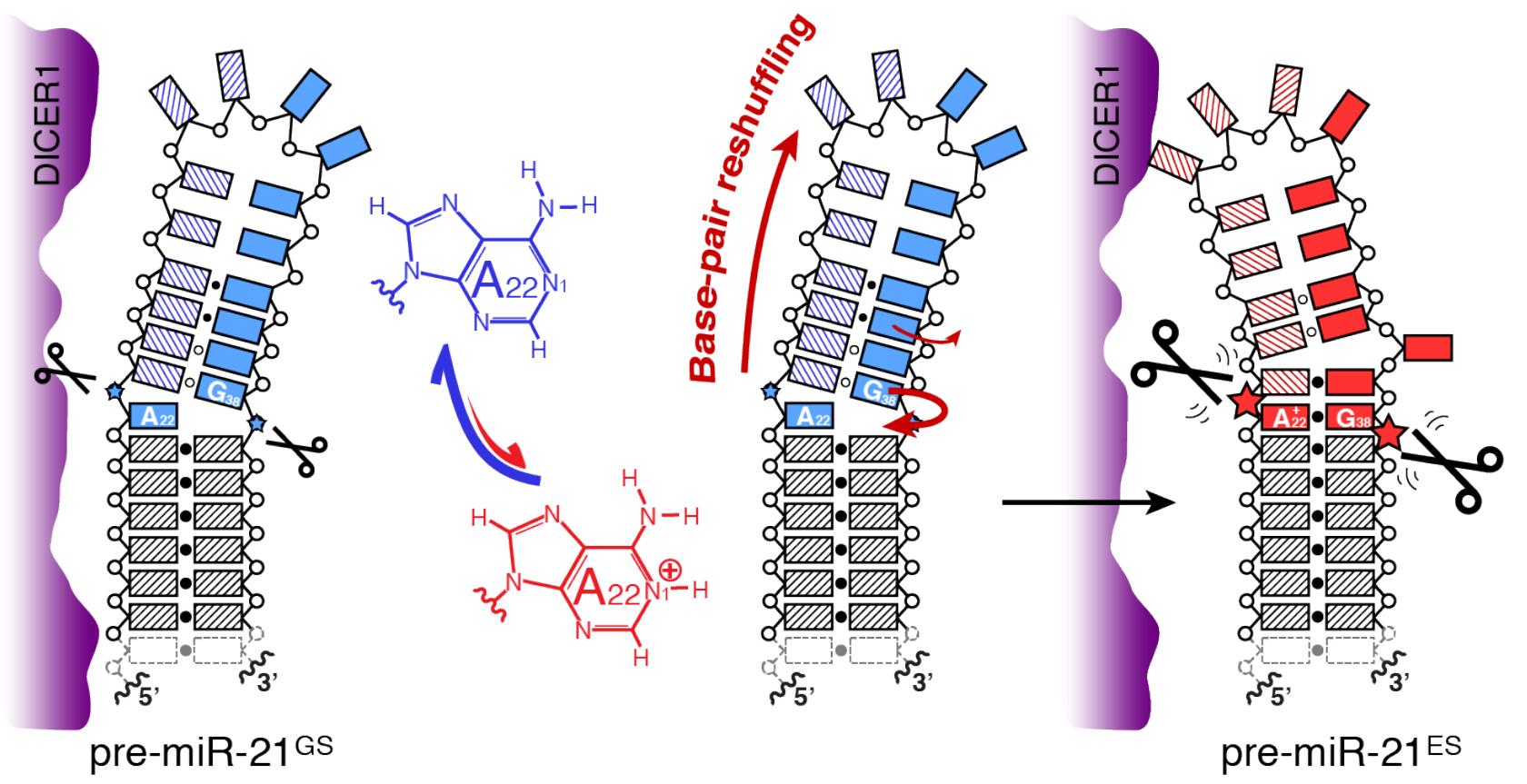

